\title{
Manipulative imagination: how to move things around in mathematics*
}

\author{
Valeria GIARDino
}

Received: $23 / 05 / 2017$

Final version: $08 / 04 / 2018$

BIBLID 0495-4548(2018)33:2p.345-360

DOI: $10.1387 /$ theoria. 17871

ABSTRACT: In the first part of the article, a semiotic reading of the embodied approach to mathematics will be presented. From this perspective, the role of the sensorimotor in mathematics will be considered, by looking at some work in experimental psychology on the segmentation of formulas and at an analysis of the practice of topology as involving manipulative imagination. According to the proposed view, representations in mathematics are cognitive tools whose functioning depends on pre-existing cognitive abilities and specific training. In the second part of the paper, the view of cognitive tools as props in games of "make-believe" will be discussed; to better specify this claim, the notion of affordance will be explored in its possible extension from concrete objects to representations.

Keywords: embodied cognition, embodied mathematics, cognitive tools, manipulative imagination, games of "make-believe", affordances.

RESUMEN: En la primera parte del artículo, se presenta una lectura semiótica del enfoque corporeizado de las matemáticas. Desde esta perspectiva, se considera el papel de lo sensoriomotor en matemática, considerando algunos trabajos en psicología experimental sobre la segmentación de fórmulas y el análisis de la práctica de la topología en términos de imaginación manipulativa. De acuerdo con la perspectiva propuesta, las representaciones en matemáticas son herramientas cognitivas cuyo funcionamiento depende de capacidades cognitivas pre-existentes y entrenamiento específico. En la segunda parte del artículo, se discute la visión de las herramientas cognitivas como 'apoyos' o 'puntales' (props) en juegos de como-si; para especificar mejor esta afirmación se explora la noción de 'affordance' en su posible extensión de objetos concretos a representaciones.

Palabras clave: cognición corporeizada, matemática corporeizada, herramientas cognitivas, imaginación manipulativa, juegos de como-si, 'affordances'

\section{Introduction: how cognition (and mathematics) gets embodied}

Core knowledge representations in cognition are amodal data structures that get processed independently of the brain's modal systems devoted to perception, action and introspection. ${ }^{1}$ This claim has been standard in cognitive science; only recently, some views have tried to challenge it. Several labels besides embodied cognition (or embodiment) have been introduced in the literature to define approaches seeking to embody cognition: embedded

* I would like to thank the participants to the Workshop "From Basic Cognition to Mathematical Practices" at the University of Seville in 2016, where I delivered a first version of this article, for their questions and comments. I also thank José Ferreirós and María de Paz for their editorial work, and two anonymous referees for their useful suggestions.

1 This sentence is a paraphrase of Barsalou (1999)'s characterization of the matter. 
cognition, extended mind, enactivism, situated cognition, grounded cognition, and so on and so forth. Indeed, these views are not equivalent; however, what they have in common is that they all maintain that the standard claim is to some extent wrong: perception, action and even introspection may play a part in our cognitive processes. ${ }^{2}$

Looking back to the history of cognitive science, there are reasons to believe that the standard claim is mistaken. Already in 1990, the great psychologist Jerome Bruner warned his readers against the wrong route that the so-called cognitive revolution of the $50 \mathrm{~s} \mathrm{had}$ taken. According to him, the cognitive revolution began by focusing on the symbolic activities involved in humans' making sense of the world and of themselves; in his words, "its aim was to prompt psychology to join forces with its sister interpretative disciplines in the humanities and in the social science" (Bruner 1990, 2). However, the emphasis gradually shifted from questions about the construction of meaning to attention towards information processing, which are "profoundly different matters" (Bruner 1990, 4). In Bruner's reconstruction, "computing became the model of the mind, and in place of the concept of meaning there emerged the concept of computability [...]. So long as there was a computable program, there was 'mind'" (Bruner 1990, 6-7). Along the same line of thought, another psychologist, Lawrence Bersalou, stressed a very similar point, by claiming that after the cognitive revolution, radically new approaches to representation were developed in contrast to pre-twentieth century thinking: "modern cognitive scientists began working with representational schemes that were inherently nonperceptual. To a large extent, this shift reflected major developments outside cognitive science in logic, statistics, and computer science" (Barsalou 1999, 578). Interestingly, in Barsalou's view, the very same practices that were brought about by the cognitive revolution were so successful that they ended up becoming the focus of research, with the initial questions that set off the revolution being forgotten.

Nonetheless, the positive claims of embodied cognition views are not always that sharply defined. Let us assume that it is in fact wrong to consider cognition as disembodied. What is then the role of perception, or action, or introspection for cognition? What does the new big picture about the mind as finally —or maybe once again - embodied amount to?

An interesting suggestion is to assume a moderate approach to embodied cognitive science as proposed by Goldman (2012). In the author's view, human cognition is for the most part embodied because information obtained from proprioception - the awareness of the relative position of one's own parts of the body — and kinesthesis - the ability to feel movements of the limbs and body- happens to be often reused for other more abstract tasks. The idea is that the brain redeploys cognitive processes having different use at their origin to solve new tasks in new contexts, which might also happen to be abstract; when it comes to bodily representational codes, they appear to be very pervasive. ${ }^{3}$ This view is particularly interesting for the present purpose precisely because it aims to explain how information obtained from proprioception and kinesthesis and thus deploying previous bodily ex-

2 For a survey of such views, see Wilson (2002) or Barsalou (2008).

3 According to Goldman and de Vignemont (2009), bodily representational codes are a subset of mental codes that are primarily or fundamentally applied in forming interoceptive or directive representations of one's own bodily states and activities; their existence is a matter of empirical investigation. 
perience can be a genuine part of cognitive processes. We will go back to this issue later in the paper in dealing in particular with cognitive tools in mathematics.

So far for cognition, but what about mathematics? Can mathematics as well be considered as embodied? There is here a nice parallel to make, which is not surprising since mathematics is a very important and ancient human activity that has been more or less stable across centuries. Recently, a plethora of studies are inscribed under the label of embodied mathematics (EM from now on). As for cognition, the standard assumption is very clear. According to a common claim, mathematics is an abstract science dealing with abstract objects that are occasionally described by signs but do not share any properties with the ordinary objects that an agent interacts with every day. EM views question the existence of such a sharp distinction between the concrete world on the one hand and abstract knowledge on the other; in fact, even when working with supposedly abstract objects, humans seem to recruit some competences and abilities that are typical of their relation with the world (the parallel with a possibly moderate approach to embodied cognition is evident here). However, also in the case of embodied mathematics, the implications of the positive claims are not that straightforward. Let us accept that the standard approach to mathematics is wrong, and previous and more spontaneous competences as developed in our interaction with the concrete world matter for doing mathematics. How does it happen? How can mathematics show itself to be in fact an embodied cognitive activity?

I propose to distinguish here between two different readings of EM: the conceptual reading and the semiotic reading. In saying that these readings are not the same, I am not claiming that they are incompatible, since there are some commonalities; still, they represent two distinct points of view regarding how to consider mathematics as embodied. In the conceptual reading, mathematical notions are conceptualized by means of grounding metaphors that are based on bodily experience; in the semiotic reading, mathematics makes use of signs that are to be perceived and acted upon (and thus sensorimotor considerations may matter). In my view, the second reading is more promising, and I will devote the rest of the paper to it. However, I will first discuss the conceptual reading so as to clarify its limits.

For what concerns the conceptual reading of EM, I refer to the by now classic work by Lakoff and Núñez (2000). Building on previous research about language (Lakoff and Johnson, 1980), the authors claim that abstract scientific concepts as well as ordinary ones are understood in terms of metaphors. To clarify, everyone - no matter if being an expert or not- would normally say that natural numbers "grow" indefinitely, or that points "lay" on a line, or that functions "move" to 0 and so on and so forth, even if it is certainly not the case that numbers literally grow bigger, or points lay on anything, or functions move anywhere. According to the authors, this is explained by the main cognitive mechanisms that are at stake when it comes to conceptualizing mathematical objects: conceptual metaphors and conceptual blending; ${ }^{4}$ in both cases, the source is bodily experience. A discussed case of conceptual metaphor is the image-schema of sets as containers. In order to understand what sets are, an agent builds the image-schema based on the metaphor according to which sets are containers: like containers, they have an interior, a boundary, and an exterior; moreo-

4 A conceptual blending allows connecting different domains through a metaphor so as to obtain a third one that is a projection of both. 
ver, the elements of the set are analogous to what is inside the container. Lakoff and Núñez claim that metaphors such as this are grounding metaphors, frequently only implicit, used to conceptualize the mathematical notion of set as determined by ZFC (the ZermeloFraenkel axioms together with the axiom of choice).

However, this reading of EM has some limits, as Schlimm (2013) has recently pointed out. According to him, Lakoff and Núñez's work surely deserves philosophical attention, because it has been the first comprehensive account of mathematical cognition that also addresses advanced mathematics and its history: mathematical ideas should be analyzed so as to provide a cognitive science theory that is about mathematics as a whole and not only about elementary mathematics. Nonetheless, a discussion from a perspective taking mathematical practice and the history of mathematics seriously into account is needed. To go back to the metaphor of sets as containers, Lakoff and Núñez suggest that there exists an "intuitive pre-mathematical notion of classes," which are "usually" and "normally" conceptualized as container schemas, and that this would be "our natural, everyday unconscious conceptual metaphor for what a class is" (Lakoff and Núñez 2000, 122-123, quoted in Schlimm 2013). However, according to Schlimm, even if it is true that the metaphor of sets as containers is found on the first pages of some introductory textbooks, later it gets quickly discarded. Moreover, mathematical practice is characterized by a back and forth of various intellectual activities: a vague metaphor can surely suggest one idea, but then a hunch or an accidental feature of the chosen notation would suggest another (we will go back to the issue of accidental features of the notation when discussing the semiotic reading of EM). In the history of a notion such as that of set, further elaborations are given by trials and errors and possibly also as a result of the interactions with other mathematicians, thus leading in turn to more sophisticated metaphors, new notations, and so on and so forth. If one wants to look at these things from the perspective of a philosophy of mathematical practice, a more fine-grained and nuanced analysis of the cognitive processes involved is needed. For example, textbook mathematics and research mathematics should be carefully distinguished (and such distinction discussed and motivated). As Schlimm sums up, such an approach should lead to a historically informed cognitive science of mathematics and a cognitive history of mathematics as it is actually practiced.

The conceptual reading of EM seems thus to be too exclusive - when it comes to mathematical conceptualization, more than one intellectual activity needs to be considered - and too strong - there is no guarantee that all mathematical concepts might be embodied this way. In the next section, I will define the semiotic reading of EM and give arguments in favor of its greater plausibility.

\section{The semiotic reading of EM: a role for the sensorimotor in mathematics}

In the semiotic reading of EM, the attention is devoted to the use of signs in mathematics, by considering whether perception or action might play a role in reasoning with them. In the following, I will present two cases in which perception and action appear to be relevant for mathematical reasoning: parsing a notational form and the practice of relying on knot diagrams in topology. 


\subsection{PARsing a notational Form}

In an interesting experimental study, Landy and Goldstone (2007) considered how physical layout might affect the segmentation of simple equations. As everyone with little competence in algebra knows, when reading a notational form, one has to segment it into its formal components; for example, a formula displaying additions and multiplication has to be parsed in such a way that multiplication is performed before addition. This is a difficult albeit routine part of mathematical reasoning. From the point of view of the standard account of mathematics, this segmentation is cognitively executed through the mere application of formal rules to individual notational symbols. According to the authors, this brings to the "appealing and tempting assumption" (Landy and Goldstone 2007, 721) that the cognitive agent - the parser- trivially extracts abstract symbol sequences from physical notations. However, to challenge this claim, they ask participants to evaluate the validity of equations containing multiplications and additions, to which visual cues such as spacing, lines, or circles have been added. The hypothesis is that such signals would influence the application of perceptual grouping mechanisms and consequently the participants' capacity for symbolic reasoning (see Figure 1).

$$
\begin{aligned}
& a+b * c+d=c+d * a+b \\
& a+b * c+d=c+d * a+b
\end{aligned}
$$

Figure 1

An example of a manipulation where spaces are added between the two factors to multiply; in this case, the visual grouping is not in line with the correct order of the operators

The results show that validity-judgments are more likely to be correct if visual groupings are in line with valid operator precedence (multiplication coming before addition); the physical layout of the equation seems thus to have an influence on performance. Of course, there is a trivial sense in which the physical layout would matter. For example, the characters of a formula can be written so small that it would not be possible for the human eye to decipher them and as a consequence to access the meaning of the formula. However, what is at stake here is less trivial: the results of the experiment show that signs happen to be targets for spontaneous sensorimotor mechanisms, analogously to other kinds of percepts. However, since signs are not simply objects but objects that are used to refer to something else, in this case to some algebraic relations, the fact that they become such targets may have an influence on our capacity to access their meaning: the manipulations applied by the authors happen to facilitate or vice versa to hinder the application of formal rules, thus showing that the layout of the formula may have an impact on its parsing. The control of such grouping mechanisms matters for the effective design of the formula and for its success in making mathematical content and operations available.

Based on these and similar results, in a more recent paper, Landy, Allen and Zednik (2014) put forward a Perceptual Manipulations Theory, according to which most of symbolic reasoning emerges from the ways in which notational formalisms are perceived and manipulated; in their view, notations serve as targets for powerful perceptual and sensorimotor systems. Other scholars as well have underlined the importance of perception and action for reading a mathematical notation. For example, Kellman, Massey and Sona (2010) 
explicitly talk of perceptual learning in mathematics, referring to the improvements that are produced by experience in the extraction of information. This would open the way to new strategies for teaching mathematics: in line with these findings, students should be trained in recognizing algebraic expressions by using standard perceptual learning techniques and this might lead to lasting gains in equation reading and comprehension, as well as in algebraic problem-solving. This is not to say that mathematical reasoning only involves perception, or that the possible actions on the notation are driven only by perceptual groupings, but to point at a crucial aspect of mathematical reasoning that has been neglected by standard accounts and that adds up to more complex cognitive and symbolic processes.

\subsection{EPistemic ACtions AND MANipUlative imagination (IN TOPOlOGy)}

The second example for a possible role for perception and action in mathematics concerns some previous work with De Toffoli on the practice of topology, a part of geometry dealing with those properties of space that remain unchanged under continuous deformations (one can think of topology as "rubber sheet geometry"). In particular, we took into account knot theory, a branch of topology that studies the properties of topological knots, and the practice of reasoning by means of knot diagrams (De Toffoli and Giardino 2014).

A topological knot is an embedding of a circle in 3-dimensional Euclidean space. A novice can think of topological knots as an abstraction of physical knots, where their ends are glued and their metric proprieties are not relevant: what matters is only their "knottedness", that is, the way in which they are knotted (or not). In order to study topological knots, mathematicians rely on knot diagrams, which are projections of topological knots following some specific constraints (for example, the information at crossings must be preserved) (see Figure 2).

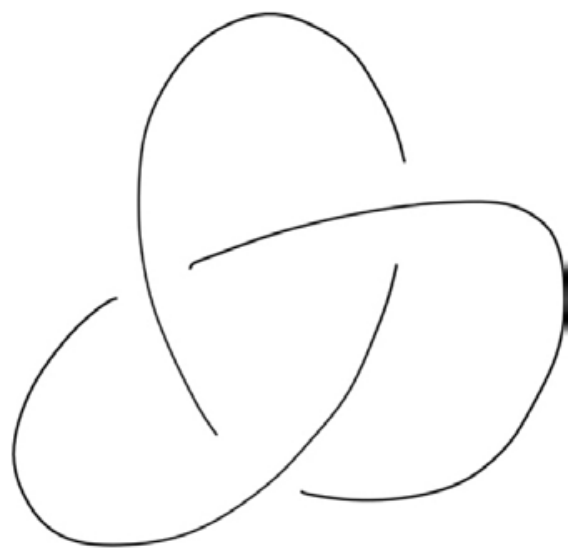

Figure 2

A knot diagram of the trefoil knot

In our study, we argued that knot diagrams are not only visual illustrations but dynamic tools: when looking at them, experts learn to perceive their possible moves, and thanks 
to that they are able to infer something about the properties of the knot. To clarify, we claimed that such diagrams trigger in the agents a specific form of imagination that we defined manipulative: experts have learnt how to perform or how to imagine performing some actions on the diagrams. For example, by looking at the diagram in Figure 2, one can imagine picking one of the threads and pull it down, so as to see that this move would not change the knottedness of the knot (it corresponds to a continuous transformation). This case shows the plausibility of the idea of a moderate embodied approach to cognition, since some kind of bodily experience seems to be redeployed when relating to more abstract tasks such as working with a topological knot. However, such competences, originating in our relation with concrete objects, are modulated by expertise. In fact, not all the possible conceivable changes of the diagram would be permitted: only some of these actions are allowed by the practice, that is, they are recognized as meaningful in relation to the specific mathematical context. Moreover, these actions can become epistemic, that means that they might lead to new inferences about some mathematical properties of the topological knot.

The term "epistemic actions" is taken from the study presented by Kirsh and Maglio (1994) on the performance of Tetris players. ${ }^{5}$ In their observations, the authors noticed that players act on the pieces of the game not only to directly achieve the final goal but also to understand what the best move will be. As a consequence, they distinguished between pragmatic actions, which aim to bring the agent closer to their physical goal, and epistemic actions, which use world to improve cognition: they are "physical actions that make mental computation easier" (Kirsh and Maglio 1994, 514) and are performed outside on the available physical objects, with the result of enhancing inference and reasoning. Despite the very elementary context of a computer game, the idea can be extended to mathematical tools, such as knot diagrams. Some transformations that are allowed by the practice can be imagined on the diagram or actually performed by rewriting it, and this would bring to experimentation and to the definition of new inferences and new results.

In the last section of the article, I will go back to a more precise definition of these actions.

\subsection{ONE CLAIM AND TWO RESEARCH DIRECTIONS}

In view of what has been said so far, the semiotic reading of EM seems to put forward one claim and two research directions. The claim is that the functioning of cognitive tools in mathematics - diagrams, notation or writing, if intended in a broad sense - and their influence on the inferences and possibly on the obtained results is crucial in mathematics. ${ }^{6}$

5 Tetris is a popular videogame from the 1980s, where some pieces that are shaped as geometric figures and composed of four squared blocks fall one after the other down the playing field. The objective of the game is to create a horizontal line below, composed of ten blocks presenting no gaps. Once a whole line is created, it disappears, and any block that is above the deleted line will fall down. The game is over if the stack of pieces reaches the top of the playing field; however, if a certain number of lines is cleared, then the game enters a new level (in which the pieces will go down faster). In order to create the line below to the aim of having it cleared, the player can move the pieces sideways and rotate them by 90-degree units.

6 Notice that I use here signs and cognitive tools alternatively; however, signs are a subset of cognitive tools. 
These tools are perceived and acted upon, and in this sense mathematics can be thought of as embodied: proprioception and kinesthesis are here recruited for more abstract uses for the very reason that these tools serve as targets for them.

Such a functioning of cognitive tools might lead to:

1. unintended effects of sensorimotor considerations on mathematical reasoning, which might become a source of notational artifacts; ${ }^{7}$

2. intended effects of sensorimotor considerations on mathematical reasoning, because these tools may serve as imagination-triggers.

If we consider the example above of the formula with spaces added between the elements to add or multiply, the grouping effects hinder the parsing when they are not in line with the correct groupings; therefore, the signs happen to be targets of sensorimotor considerations, but they are not intended to be as such. On the contrary, the physical features of knot diagrams are useful and effective precisely because they trigger manipulative imagination in such a way that it enhances reasoning and inference. Such a distinction defines two complementary research directions in looking at case studies and possibly in defining experimental settings where such effects can be evaluated. This of course runs counter to the standard claim about mathematics, since the physical features of the signs may indeed have an influence on mathematical reasoning.

\section{How to characterize manipulative imagination and epistemic actions}

In this section, I will try to specify more my claim about manipulative imagination as triggered by some particular representations. Firstly, I will look at a possible general framework about imagination; secondly, I will focus on the epistemic actions that are the results of this form of imagination.

\subsection{Cognitive tools as props in games of MAKe-Believe}

What possible general framework about the workings of imagination will be appropriate for mathematics? In this section, I will consider an application of Kendall Walton (1990)'s theory about the nature of representations to cognitive tools in mathematics.

According to Walton's "make-believe" theory, representations are props in games of make-believe, that is, they are supports for imagination analogously to objects such as dolls or toy tracks in the games played by children. To clarify, if a child imagines that a doll is a baby, depending on what happens to the doll, she will imagine various things happening to the baby as well: cradling the doll would be cradling the baby, or singing a lullaby to the doll would be singing it to the baby. However, there are rules by which props prescribe imaginings, which are called principles of generation. Such principles are normative: they say what participants should imagine, given the state of the props, if they are playing the game correctly. However, these principles are often implicit and not explicitly stated. Imaginings

7 As in experimental science, "artifact" is intended here as error resulting from flaws in the deployed technique or equipment —or, in this case, in a particular tool. 
that are prescribed within the game are fictional and such a normative approach preserves an account of truth in the fiction.

Adam Toon (2010a, 2010b, 2011) has recently proposed to rely on Walton's view in philosophy of science, in particular to give an account of the use of physical as well as theoretical models (these latter being aimed at constructing a simplified or idealized version of some system). In most cases, models are false of the system they want to represent: even if they look like a description of some actual, concrete object, no such system would satisfy this description. However, in Toon's reconstruction, they are still representations because, analogously to dolls or toy trucks for children, they serve as props in games of make-believe. In the case of a physical model, for example in chemistry, scientists do imagine plastic balls and connecting rods to be molecules in much the same way that children imagine a doll to be a baby; in a theoretical model, for example the model of a bouncing spring as a simple harmonic oscillator used to predict the motion of a bob bouncing on the end of a spring, scientists would of course realize that such a description is false of the world (there is no reference to air resistance or to the mass of the bob), but they would still be required to imagine things as the model prescribes, for example, that the bob is a point mass or that the spring exerts a linear restoring force, and so on and so forth. Also in the case of models, the principles of generations, in most cases implicit, are normative: the scientist knows that she cannot misinterpret the model. Moreover, there must be an understanding among users that various imaginings are prescribed depending on the features of the model: the imaginings about its object as prescribed by the model are conditional on those properties. Such an approach may also address the ontological difficulties that are posed by theoretical modeling. In fact, in Toon's interpretation, to claim that a model is a representation in Walton's sense does not amount to assume anything about the existence of the entities it invokes. Models function as props in games of make-believe and need not prescribe imaginings about any actual system; however, they are still representational because they prescribe imaginings about the modeled system and make propositions about it fictional. Such a view does not incur any 'ontological commitments': Walton's theory is antirealist with regard to fictional entities. ${ }^{8}$

It seems then that Walton's "make-believe" theory can be useful for an account of models in science. What about cognitive tools in mathematics? Can they be considered as well as props in games of make-believe? To some extent, this view seems very plausible.

Take again the case of knot diagrams, and assume that they are props in make-believe games. Indeed, mathematicians do imagine knot diagrams to be the knots-as it is evident in the ambiguous way they speak alternatively of "knots" or "knot diagrams" - in much the same way that children imagine a doll to be a baby or toy tracks to be real tracks; furthermore, they imagine themselves as viewing and manipulating knots and sometimes even 'living' in the depicted topological object-therefore, not only they are required to imagine things about the knots but also they learn what further propositions these imaginings

8 Frigg (2010) also suggests that the descriptions presented in theoretical modeling should be understood as props in Walton's sense; however, in his view these descriptions prescribe the user to imagine 'model systems' understood as imagined physical systems, that is, as hypothetical entities. Toon criticizes Frigg's view by claiming that it is not clear how this would not incur any ontological commitment. 
entail. For knot diagrams as for models, principles of generations are normative: the mathematician knows that she cannot misinterpret the diagram and that only some of all the possible actions that can be performed on them are meaningful in relation to the practice. However, these principles are in most cases implicit and not explicitly defined. This approach may also be of help to deal with ontological issues regarding the object of mathematics. In fact, a Walton-inspired view of cognitive tools as props in games of make-believe is antirealist: the propositions that can be inferred by the representation are considered as fictional, but this does not imply taking any stance about the existence of the represented objects.

However, some difficulties emerge. First, this approach seems to apply very easily to representations such as knot diagrams but less conveniently to other kinds of cognitive tools such as the equations that were presented in Landy and Goldstone's study. Can a formula be intended as a prop for some game of make-believe? Concerning this question, it seems to me that there are two possible ways out. First, one could think that it is simply not possible to give a unified account for cognitive tools in mathematics as a whole; for example, further distinctions such as between iconic and symbolic representations should be introduced. However, there is an alternative, which is to reply positively to the question whether an equation can serve as well as a prop, and to claim that also when working with formulas mathematicians are required to have some imaginings, for example they form propositions about algebraic relations or imagine applying transformations on them. The distinction between knot diagrams and formulas can be thought of as not being so distant from that between physical models and theoretical models in science: in all cases, these tools serve as props to prescribe imaginings; for knot diagrams and physical models, their physical features take part in the imaginings; for formulas and theoretical models, the required imaginings involve relations that are expressed by the signs displayed in the equations. In any case, the fact that they are props prescribing imaginings does not imply the existence of the objects they represent.

However, another difficulty in assuming a Waltonian approach to cognitive tools in mathematics concerns the definition of principles of generations in this specific context. In the case of representational art, principles of generations, which are often implicit, depend on conventions and cultural constraints. For models in science, they may even more importantly vary from case to case. What about cognitive tools in mathematics?

Before trying to reply to this question, let me sum up what I have got so far. I proposed that cognitive tools in mathematics can be considered as representations in Walton's terms, analogously to what Toon suggests for models in science: they serve as props for games of make-believe. However, in order for them to be as such, the users must agree on the various prescribed imaginings depending on the physical features of the props, despite the fact that principles of generation are in most cases implicit and most likely vary from case to case. In order to clarify how these principles are characterized, in the next section I will focus on the epistemic actions that these tools make accessible for the competent user.

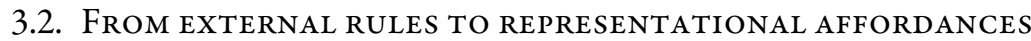

Cognitive tools are external and material tools that are used in cognitive tasks. In the previous section, I claimed that they are props for imagining, but their use has to be constrained in order to be effective. How are these constraints defined? 
In a relatively old paper, Zhang and Norman (1994) proposed that many cognitive tasks require the processing of information to be distributed across the internal mind and the external environment; in other words, such tasks are distributed between internal and external representations, which, taken together, define the abstract structure of the problem. Internal representations are in the mind: they may take the form of propositions, schemas, mental images, connectionist networks, and so on and so forth. External representations are instead in the world: they might be physical symbols - no matter if written symbols or beads of abacuses - external rules, constraints, or relations embedded in physical configurations. Think for example of the spatial relations between written digits, or of the visual and spatial layouts of diagrams, or of physical constraints in abacuses, and so on and so forth. In any distributed cognitive task, there are in general one or more internal and external representations involved.

As a case study, the authors consider the task of the Tower of Hanoi. ${ }^{9}$ This cognitive task involves a problem space that is composed by all the possible states and moves constrained by the rules. Two factors contribute to difficulty: working memory and problem structure. In the experiment, participants are presented with several versions of the task, involving each time a variation of internal and external rules. As the authors explain, internal rules are "memorized rules that are explicitly stated as written propositions in the instructions of the experiment", while external rules "are not stated in any form in the instructions but are the constraints that are embedded in or implied by physical configurations and can be perceived and followed without being explicitly formulated" (93). The results show that the more the rules are distributed in the external representation, the easier the problem gets and the fewer errors are made. The authors' hypothesis is that an excessive load of working memory causes internal errors, while external rules are 'built-in' in the external representations, that is, they are either perceptually available or physically constrained. To clarify, the disks are perceived as bigger or smaller and this reduces memory loads, for example when applying the rule that only the bigger disk on a pole can be transferred to another pole.

If this is the case, do cognitive tools in mathematics embody external rules analogously to the disks in the Tower of Hanoi task? Some external rules might be built-in in them, so that they become perceptually available or physically constrained. It is true indeed that mathematicians do act upon the cognitive tools at their disposal, of which they have to consider the physical features in order to apply transformations to them; it seems therefore that some external rules might really be built-in in them, because they are determined by their material constraints. ${ }^{10}$

9 In this task, the participant is presented with three rods; on the first rod, three disks of different size are arranged in a stack, with the bigger disk being at the bottom and the smaller disk at the top. The objective of the puzzle is to move the entire stack to another rod. In the standard version of the task, this must be done by obeying the following simple rules: (i) only one disk can be moved at a time; (ii) each move consists of taking the upper disk from one of the stacks and placing it on top of another stack, i.e. a disk can only be moved if it is the uppermost disk on a stack; (iii) no disk may be placed on top of a smaller disk. Note that, in this standard case, rule (i) and rule (ii) are internal rules, while rule (iii) is external.

${ }^{10}$ From this point of view, according to Manders (2008)'s definition, co-exact properties in Euclid proofs could be interpreted as defining external rules, since they identify the physical features of the diagram that would not be changed after deforming the diagram, and are thus "built-in" in it. 
However, this approach has some limits. The first problem is that in the case of the Tower of Hanoi task, external rules are considered in relation to external concrete objects with which one directly interacts - the disks - while mathematical objects such as knot diagrams, because of their nature of representations, require a particular reading in order to understand to which objects they refer to. Not all the material features of the tool but only a selection of them are relevant, depending on the correct interpretation as defined by the practice. In fact, a representation has to be correctly interpreted, and the correct interpretation requires background knowledge and/or the understanding of the accompanying text. This leads to a second and even more serious difficulty, which is that if interpretation matters so much, then in the case of cognitive tools it is very hard to draw a sharp line between internal and external rules. How much of what is claimed in Zhang and Norman's paper about distributed cognitive tasks can be then applied to external 'representational' objects such as signs in mathematics?

Despite these limitations, the very authors seem to suggest a possible way out, by mentioning that external rules provide affordances. To clarify, the term "affordances" refers to the actionable properties between the environment and an agent (a person or an animal) and was introduced by James J. Gibson (1979) when presenting his ecological approach to visual perception. Affordances are what the environment offers the animal, "what it provides or furnishes, either for good or ill. The verb to afford is found in the dictionary, but the noun affordance is not. I have made it up. I mean by it something that refers to both the environment and the animal in a way that no existing term does. It implies the complementarity of the animal and the environment" (Gibson 1979, 127).

To give an example, a surface that is horizontal, flat, extended and rigid affords support. The interesting point here is that the world of physics is not as important for us as our environment of affordances. Of course, a surface would afford support because it possesses these specific and appropriate physical properties, but what counts for us is the affordance it provides. To focus on the notion of affordance means to avoid a sharp distinction between a subject on the one hand and an object on the other hand. As Gibson explains, an affordance is "neither an objective property nor a subjective property; or it is both if you like. An affordance cuts across the dichotomy of subjective-objective and helps us to understand its inadequacy. It is equally a fact of the environment and a fact of behavior. It is both physical and psychical, yet neither. An affordance points both ways, to the environment and to the observer" (Gibson 1979, 129, emphasis added). If this is the case, then there is no need to distinguish sharply between internal and external rules; thanks to some physical features, concrete objects afford some actions and not others; by their affordances, such objects might influence the behavior of the agent and, in this particular case, facilitate or not the cognitive task. However, affordances in the context of the Tower of Hanoi task are still pertaining to external concrete objects such as the disks that are part of a problem-solving task. What about affordances offered by cognitive tools?

Gibson discusses the natural environment; however, artifacts may be designed as offering particular affordances, with the intention of triggering some particular behavior. Think for example of a door with a handle. Such a feature, the handle, affords pulling; however, if the door only opens by pushing, then the affordance would not be in accordance with what it offers the agent's behavior. As a consequence, one might think in terms of good design — the door has a handle and opens if one pulls it-, and bad design — the door has a handle and opens if one pushes it. If the notion of affordance can be extended from the natural 
environment to artifacts, would it possible to extend it even further, from artifacts to representational tools such as notation or diagrams in mathematics? Cognitive tools do afford some actions on them. In their case, we can think in terms of representational affordances. Of course, not all possible actions that are afforded by the tool are also mathematically meaningful: only a selection of them corresponds to a legitimate and therefore an epistemic operation, because of other constraints depending on their specific function or the specific practice for which these tools are introduced. ${ }^{11}$ Here as well it is possible to think in terms of good or bad design: if the tool is well-designed, epistemic actions should be easily available and allow for relevant inferences.

To give an example, the knot diagram in Figure 2 shows occlusions at crossings; thanks to grouping laws of perception such as good continuation, ${ }^{12}$ these occlusions are directly interpreted as threads passing one under the other. Therefore, these diagrams trigger imagination by affording transformations on them that are immediately available, such as imagining pulling down one or more threads in sequence so as to obtain equivalent knot diagrams. Other representations containing the same information but presenting different physical features, for example by using dots to indicate a crossing as in Figure 3, would not offer the same affordances and might hinder relevant transformations. ${ }^{13}$

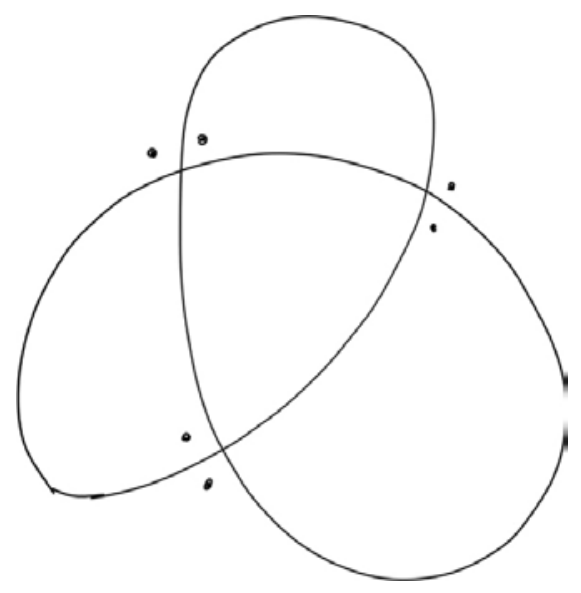

Figure 3

An alternative knot diagram of the trefoil knot

${ }^{11}$ I take the term "legitimate operations" from Alexander (1923, 94).

12 Good continuation is one of the Gestalt laws of groupings, thanks to which in an intersection between two or more lines one of which is interrupted, people tend to perceive each line as a single uninterrupted one.

13 Alexander (1928) uses these diagrams, and explains as follows: "At each crossing point, two of the four corners will be dotted to indicate which of the two branches through the crossing point is to be thought of as the one passing under, or behind the other. The convention will be to place the dots in such a manner that an insect crawling in the positive sense along the «lower» branch through a crossing point would always have the two dotted corners on its left" (Alexander 1928, 276-277). 
However, another important feature of affordances for cognitive tools is that they are highly context-dependent. The same notation or diagram might afford different actions and transformations when having different functions - for example of discovery or of proof-, or across different disciplines - think of two (dotted) parallel lines in the context of Euclidean or projective geometry. Therefore, I will distinguish between perceptual representational affordances and context-dependent representational affordances. On the one hand, cognitive tools spontaneously afford some actions because of the functioning of our cognitive system: for example, it is thanks to good continuation, which applies to other percept as well, that one sees the threads in a knot diagram as uninterrupted lines even if they are interrupted in the diagram. On the other hand, cognitive tools also afford actions that are determined by expertise: this second kind of affordances defines the principles of generation that are at each time at stake and determine what is correct and what is not. However, these principles are still implicit and depend on the specific make-believe games that the user is engaged in.

If this is the case, then two general consequences can be drawn. First, a design principle can be defined, according to which it is commendable to prevent the perceptual representational affordances of cognitive tools from being sources of errors. This is the case of the manipulations applied to the equations in Landy and Goldstone's experiment: adding spaces or lines trigger features of our cognitive systems, such as perceptual groupings, and this may lead to unintended and in some cases damaging effects of sensorimotor considerations on mathematical reasoning. On the contrary, it would be very convenient to exploit perceptual representational affordances to facilitate the relevant and correct operations and make them correspond to legitimate actions that are meaningful also in the mathematical context. This is the case of displaying occlusions in a knot diagram, so as to trigger continuous transformations. Second, the context-dependence of some representational affordances has to be recognized and seriously taken into account: different contexts or functions can make different context-dependent representational affordances emerge, but these affordances are still conditional on the physical features of the tool and keep interacting with the perceptual representational ones. One might also envisage cases where a perceptual affordance is so much forcing on us, that it contributes at identifying new meaningful and epistemic actions (think for example of the potential role of symmetries, to which we very spontaneously attend).

To sum up, my proposal is that cognitive tools in mathematics are props for manipulative imagination, and they are well designed when they spontaneously afford the meaningful transformations that lead experts - who are able to correctly interpret the representation and who know the implicit principles of generation for the specific game of make believe they are playing - to epistemic and inferential actions. The introduction of such cognitive tools presents several advantages. Firstly, they are material objects and therefore in order to be used they recruit proprioception and kinesthesis, which are now deployed in a new more abstract cognitive context. There is thus a - semiotic- sense in which mathematics can be (moderately) embodied. Cognitive tools offer representational affordances, some of which directly depend on the perception of some of the physical features of the tool (perceptual representational affordances), and others that more strongly require interpretation (context-dependent representational affordances). Secondly, if representational affordances are constrained by the practice, they facilitate common imaginings: users understand that various imaginings are prescribed depending on the physical features of the tools. 
Thirdly, by referring to the notion of representational affordance, it is possible to bypass the problematic distinction between internal - propositional-, and external — built-in in the material object - rules. Finally, both unintended and intended effects of sensorimotor considerations in the use of several mathematical signs can be reframed as having to do with bad versus good design.

\section{Conclusions}

To recapitulate, in this article I have proposed a semiotic reading of EM, according to which mathematics makes use of signs that are to be perceived and acted upon (and thus sensorimotor considerations may matter). My attempt is to make sense and clarify the functioning of the practice involving the use of cognitive tools such as notation or diagrams.

The theoretical framework that I have sketched here refers to the activity of make-believe as based on props that offer some specific affordances, in line with the practice: from this perspective, an accommodated version of Walton's theory of representation meets Gibson's view according to which affordances are provided by the environment -in this case, a very special kind of environment in which mathematicians move, which is populated by the diverse representational cognitive tools that are at their disposal. Specific actions on cognitive tools happen to be crucial for mathematical reasoning, and cognitive tools trigger these actions by offering representational affordances; as a consequence, they promote imagination as make-believe. These actions - just imagined or carried out by rewriting - conform to some constraints: by "moving things around", we actually perform on the material signs legitimate and therefore meaningful operations, thus enhancing mathematical reasoning.

\section{REFERENCES}

Alexander, James W. 1923. A Lemma on systems of knotted curves. Proceedings of the National Academy of Sciences of the United States of America 9/3: 93-95.

-. 1928. Topological invariants of knots and links. Transactions of the American Mathematical Society 30/2: 275-306.

Barsalou, Lawrence W. 2008 Grounded cognition. Annu. Rev. Psychol. 59, 617-45.

-. 1999. Perceptual symbol systems. Behavioral and Brain Sciences 22: 577-660.

Bruner, Jerome. 1990. Acts of meaning, Cambridge, MA: Harvard University Press.

De Cruz, Helen and Johan De Smedt. 2013. Mathematical symbols as epistemic actions. Synthese 190: 3-19.

Frigg, Roman. 2010. Models and fiction. Synthese 172: 251-268.

Gibson, James J. 1979. The ecological approach to visual perception. Boston: Houghton Mifflin.

Goldman, Alvin. 2012. A moderate approach to embodied cognitive science. The Review of Philosophy and Psychology 3/1:71-88.

Goldman, A. and Frederique de Vignemont. 2009. Is social cognition embodied? Trends in Cognitive Sciences 13/4: 154-159.

Kellman, Philip J., Christine M. Massey, and Ji Y. Sona. 2010. Perceptual learning modules in mathematics: Enhancing students' pattern recognition, structure ex- traction, and fluency. Topics in Cognitive Science 2/2:285-305. 
Kirsh D. and Paul Maglio. 1994. On distinguishing epistemic from pragmatic action. Cognitive Science 18/4:513-549.

Lakoff, George P. and Mark L. Johnson. 1980. Metaphors we live by. Chicago: University of Chicago Press.

Lakoff, George P. and Rafael Núnez. 2000. Where mathematics come from: How the embodied mind brings mathematics into being. New York: Basic Books.

Landy, David, Colin Allen, and Carlos Zednik. 2014. A perceptual account of symbolic reasoning. Frontiers in Psychology 5, 275.

Landy, David, and R. L. Goldstone. 2007. How abstract is symbolic thought? Journal of Experimental Psychology: Learning, Memory, and Cognition 33/4: 720-733.

Macbeth, Danielle. (2012) Seeing how it goes: Paper-and-pencil reasoning in mathematical practice. Philosophia Mathematica 3/20: 58-85.

Manders, Kenneth. (2008). The Euclidean diagram. In P. Mancosu, ed, The Philosophy of Mathematical Practice, 80-133. Oxford: Oxford University Press.

Netz, Reviel. 1999. The shaping of deduction in Greek mathematics: A study of cognitive history. Cambridge: Cambridge University Press.

Schlimm, Dirk. 2013. Conceptual metaphors and mathematical practice: On cognitive studies of historical developments in mathematics. Topics in Cognitive Science 5/2: 283-298.

Toon, Adam. 2010a. The ontology of theoretical modelling: Models as make-believe. Synthese 172/2: 301315.

-. 2010b. Models as make-believe. In Frigg R. and M. Hunter eds., Beyond mimesis and convention: Representation in art and science, 71-96. Dordrecht: Springer.

-. 2011. Playing with molecules. Studies in History and Philosophy of Science Part A 42/4: 580-589.

Walton, Kendall. 1990. Mimesis as make-believe: On the foundations of the representational arts. Cambridge, Mass.: Harvard University Press.

Wilson, Margaret. 2002. Six views of embodied cognition. Psychonomic Bulletin \& Review 9/4: 625-636.

Zhang, Jiajie and Donald A. Norman. 1994. Representations in Distributed Cognitive Tasks. Cognitive Science 18: 87-122.

Valeria Giardino is Researcher at the CNRS (Centre National de la Recherche Scientifique), affiliated to the Henri Poincaré Archives in Nancy, France. She studied Logic and Epistemology at the University of Rome La Sapienza where she also obtained her PhD. She worked as a Postdoc at the Institut Jean Nicod in Paris, at Columbia University, at the University of Seville, at the Freie University of Berlin, and at the École Normale Supérieure of Paris. Her main research interests are the philosophy of the mathematical practice, and in particular the role and the functioning of visual representations for mathematical reasoning.

Address: Archives Henri-Poincaré - Philosophie et Recherches sur les Sciences et les Technologies. UMR 7117 CNRS - Université de Lorraine (91 avenue de la Libération - BP 454. F-54001 NANCY Cedex). Email: valeria.giardino@univ-lorraine.fr 